\title{
Blood vessels, disease pathogenesis, and novel therapies
}

\author{
Gregg L. Semenza
}

Published online: 22 February 2013

(C) Springer-Verlag Berlin Heidelberg 2013

This issue of the Journal of Molecular Medicine contains four outstanding review articles by leaders in the field that summarize recent advances in endothelial biology, angiogenesis, and vascular remodeling. This remains a dynamic area of research because of the remarkably broad role that vascular changes play in all of the common causes of mortality, including heart disease, cancer, stroke, chronic lung disease, chronic kidney disease, peripheral vascular disease, and diabetes.

One area of ongoing scientific debate has concerned the criteria for identifying endothelial progenitor cells (EPCs). By definition, EPCs should give rise to endothelial cells, but the term has been used loosely to encompass many different cell types that may contribute to endothelial replacement, vascular remodeling, and reparative responses to ischemia. Yoder [1] discusses bone marrow-derived cells that display angiogenic properties and contrasts current problems regarding EPC nomenclature with the well-defined developmental hierarchy of hematopoietic stem, progenitor, and effector cells.

The regulation of blood flow in the pulmonary vasculature differs dramatically from the systemic vasculature. In response to local tissue hypoxia, systemic arterioles dilate to increase blood flow and improve oxygenation. In contrast, pulmonary arterioles constrict in response to hypoxia in order to shunt blood away from areas of the lung that are not ventilated. Whereas this is an adaptive response in the context of lobar pneumonia, it is a maladaptive response under conditions of global alveolar hypoxia, which may

\section{G. L. Semenza $(\bowtie)$}

Vascular Program, Institute for Cell Engineering; Departments of Pediatrics, Medicine, Oncology, Radiation Oncology, and Biological Chemistry; and McKusick-Nathans Institute of Genetic Medicine, Johns Hopkins University School of Medicine, Baltimore, MD 21205, USA

e-mail: gsemenza@jhmi.edu occur during residence at high altitude and in patients with chronic lung disease. Shimoda and Laurie [2] summarize recent advances in delineating the effects of chronic hypoxia on pulmonary arterial smooth muscle cells that contribute to the pathogenesis of pulmonary hypertension.

Pathological angiogenesis in the eye is the major cause of vision loss in developed countries. Campochiaro [3] summarizes the molecular mechanisms underlying ocular neovascularization in a wide variety of eye diseases and discusses potential novel strategies to treat these disorders.

Byzova and her colleagues [4] focus on the interconnections between inflammation, oxidative stress, and angiogenesis. The therapeutic implications of recent findings that implicate inflammatory cells in the development of cancer resistance to angiogenesis inhibitors are also discussed.

The principles highlighted in these four review articles have applications to many common disease states. As a result, these papers will be of broad interest to the readers of the Journal of Molecular Medicine. One principle is clear: therapies are most likely to succeed when they are built upon a strong foundation with respect to knowledge of the underlying disease pathophysiology and of the treatment's mechanism of action.

\section{References}

1. Yoder MC (2013) Endothelial progenitor cell: a blood cell by many other names may serve similar functions. J Mol Med. doi:10.1007/ s00109-013-1002-8

2. Shimoda LA, Laurie SS (2013) Vascular remodeling in pulmonary hypertension. J Mol Med. doi:10.1007/s00109-013-0998-0

3. Campochiaro PA (2013) Ocular neovascularization. J Mol Med. doi: 10.1007/s00109-013-0993-5

4. Kim YW, West XZ, Byzova TV (2013) Inflammation and oxidative stress in angiogenesis and vascular disease. J Mol Med. doi: 10.1007/s00109-013-1007-3 